\title{
Active Recognition Attempts Induce Updating of Face Memories
}

Matthew Plummer ${ }^{1}$, Robin Hellerstedt ${ }^{1,2}$, Stuart J. Gibson ${ }^{3}$, Jon S. Simons ${ }^{2}$ and Zara M.

\author{
Bergström $^{1}$ \\ ${ }^{1}$ School of Psychology, University of Kent \\ ${ }^{2}$ Department of Psychology, University of Cambridge \\ ${ }^{3}$ School of Physical Sciences, University of Kent
}

\begin{abstract}
Author note
Matthew Plummer https://orcid.org/0000-0003-1914-7750

Robin Hellerstedt https://orcid.org/0000-0002-6433-4734

Stuart Gibson https://orcid.org/0000-0002-7981-241X

Jon Simons https://orcid.org/0000-0002-7508-9084

Zara Bergström https://orcid.org/0000-0001-8794-0182
\end{abstract}

This is a manuscript draft that has not yet been peer reviewed or published in a journal (25/10/21). Data will be shared on the Open Science Framework when the manuscript is accepted for publication. We have no conflicts of interest to disclose. This research was supported by a Research Project Grant (RPG-2016-322) from the Leverhulme Trust awarded to ZB (PI), SG (Co-I) and JS (Co-I). Correspondence concerning this article should be addressed to Dr Zara Bergström, School of Psychology, University of Kent, Canterbury, Kent, CT2 7NP. Email: z.m.bergstrom@kent.ac.uk. Phone: (0)1227 827507. 


\begin{abstract}
While successful retrieval typically strengthens memories, errors made during retrieval attempts can become encoded and bias subsequent remembering. It is not clear however whether such updating occurs during recognition of faces based on their visual properties. We investigated recognition-induced updating of face memories across three experiments, by comparing the effects of active recognition attempts against two control tasks that also exposed participants to erroneous face information while they were not trying to remember. Importantly, we used computer generated facial images drawn from locations in a multidimensional "face space" to match the degree of error that was introduced by the different tasks, enabling us to isolate the role of active retrieval processes in face memory updating. Participants first learned a series of target faces. Next, target faces were shown mixed with similar distractor faces and participants either actively tried to recognize the targets, or tried to encode one of the faces, or selected the face they thought was most distinctive. We then tested participants' recognition memory for targets in a surprise final test, and measured to what extent their recognition errors on the final test were biased by their responses on the prior task. Across the three experiments, final recognition bias was significantly enhanced after active recognition attempts and was larger following recognition attempts compared to either control task. The findings extend on prior demonstrations that retrieval-induced updating occurs for semantically rich, complex memories by showing that engagement of active retrieval processes during visually-based face recognition can also induce updating.
\end{abstract}


Remembering past events can strengthen memories, increasing the likelihood that those memories will be possible to retrieve again in the future (Roediger \& Butler, 2011). Testing memory is a more effective way to enhance long-term retention than re-studying the same information, suggesting that active retrieval attempts are particularly important for learning (Roediger \& Karpicke, 2006). However, retrieval can also induce memory errors so that information that was once accurately remembered becomes distorted (Schacter et al., 2011). Previous research on human long-term memory updating has primarily focused on how recollection of complex event memories induces distortions. Here, we investigated whether visually-based recognition of human faces induces similar distortions.

Intentionally retrieving past events involves a reconstructive process that is prone to error and renders reactivated memories malleable. Long-term memory retrieval is thought to involve reactivation of dormant memory traces, allowing those memories to become updated when novel information present in the retrieval environment becomes encoded and integrated with the original memory (Dudai \& Eisenberg, 2004; Hupbach et al., 2007; Lee et al., 2017; St Jacques \& Schacter, 2013). Via this process, errors made during retrieval can distort subsequent attempts to remember. For example, when people are asked to repeatedly recall the spatial location in which they previously encountered an object, their responses on later tests are more similar to erroneous responses they made on prior tests than the original location where they first saw the object (Bridge \& Paller, 2012). Furthermore, such spatial memory distortions are enhanced when participants actively try to retrieve a location from memory, and not when they are passively cued to select a location determined by experimenters (Bridge \& Voss, 2014). Intentional attempts to retrieve a stored memory involves a cognitive state referred to as "retrieval mode" (Tulving, 1983, Rugg \& Wilding, 2000), which is thought to elicit reactivation of stored memories more effectively and more reconstructively than unintentional retrieval processes that are engaged automatically by 
reminders. Accordingly, errors made during intentional retrieval attempts should be particularly likely to become encoded into updated memories, compared to when people encounter erroneous information in other cognitive states.

Whilst prior research has demonstrated that long-term memories can change as a result of active retrieval attempts, these studies have primarily focused on situations where semantically meaningful and complex event memories are updated as a consequence of being recalled (e.g. Bridge \& Paller, 2012; Bridge \& Voss, 2014; Carpenter \& Kelly, 2012; St Jacques \& Schacter, 2013; Ye et al., 2020). It is less known whether similar updating processes are engaged during intentional recognition of stimuli that contain minimal semantic information, such as when we attempt to recognize previously seen but personally unfamiliar faces based on their visual properties. Such recognition may involve brain systems that are specialized for processing visual face information (Duchaine \& Yovel, 2015; Haxby \& Gobbini, 2011; Kanwisher et al., 1997), and may therefore potentially be differentially sensitive to updating compared to brain systems involved in memory for complex events. Furthermore, recognition of "items" (such as individual objects or faces) is thought to rely on different neurocognitive mechanisms compared to recall of complex event memories consisting of associations between multiple items and contexts (such as object-location associations). Retrieving item-context associations is thought to require recollection, which is supported by a pattern-completion mechanism in the hippocampus that reconstructs a memory by binding together different features that are stored in distributed neocortical regions (e.g. Horner et al., 2015; Rolls, 2016). However, hippocampally mediated recollection may not be necessary for recognising an item independent of its context, which may be achieved through a relatively more automatic pattern-generalisation "matching" mechanism in the adjacent medial temporal cortex, which gives rise to a feeling of familiarity for that item (Ranganath \& Ritchey, 2012; Yonelinas, 2002; although see also Bird, 2017). 
Therefore, it is unclear whether face recognition can induce memory updating, since it may not engage the reconstructive process of recollection. Addressing this issue is however important for determining the generality of memory updating across different types of memory systems and retrieval processes, and for understanding how memory distortions emerge in real world settings.

Some evidence that face recognition can induce updating comes from applied forensic contexts, where eyewitnesses of a crime may be asked to complete multiple recognition tests involving identifying a suspect's face among a line-up of foil faces. This research has found that witnesses are likely to repeatedly select the same face across multiple identification attempts even if they had falsely recognised an innocent individual at the initial identification, suggesting that initially selected faces are encoded and bias subsequent recognition attempts (see Lin et al., 2019; Steblay \& Dysart, 2016). In a related finding, people sometimes show evidence of face memory distortions after creating or viewing a 'facial composite' (a mockup image of a suspect based on eye-witness memory), causing them to mistakenly recognise the composite instead of the true face of a suspect they originally saw (e.g. Topp-Manriquez et al., 2016; although see Davis et al., 2014; Sporer et al., 2020). However, this area of research has typically focused on implications of repeated testing for real life settings, and little progress has been made in specifying the underlying cognitive processes that may be responsible for biases induced by repeated face recognition.

Another line of relevant evidence comes from research on test-induced learning ("testing effects" or "retrieval practice" effects), where it has been found that although recall tests typically lead to the largest amount of test-induced learning, recognition memory tests can also enhance later memory for tested information (Rowland, 2014). In educational settings, multiple choice tests that involve selecting between a correct answer and other plausible but incorrect alternatives can enhance later remembering of information related to 
both correct and incorrect answers (Little et al., 2012; Marsh et al., 2007). Test-induced strengthening of memories is enhanced by intentional retrieval attempts (Karpicke \& Zaromb, 2010), including on recognition tests (e.g. Gao et al., 2016; see also Liu et al., 2017). These findings show that recognition attempts can involve sufficiently "active" retrieval processing to induce memory strengthening. However, since this line of research focuses on test-induced facilitation effects for semantically meaningful memories, it is unclear to what extent those findings apply to our current research question of whether visually-based recognition of faces induces memory distortions.

Here, we report on three experiments conducted to assess whether recognition induces updating of face memories, by comparing whether errors made during active recognition attempts are more likely to lead to subsequent memory distortions than other kinds of exposure to erroneous face information. We developed a new paradigm where we controlled the extent to which recognition test faces diverged from the initially learned target faces to enable sensitive measurement and a high degree of control over recognition errors. This control was afforded by a method for generating near photo-realistic face imagery that is based on recent advances in facial composite creation (Gibson et al., 2003; Solomon et al., 2013). The method involved constructing a mathematical representation of "face space" (Valentine et al., 2016), in which a unique facial image is represented by a single point (or equivalently by a multidimensional vector). Accordingly, the similarity between any two face images can be quantified by measuring the corresponding distance between points in the face space model (Mist et al., 2015). Because these artificially generated face images were unfamiliar to participants prior to the experiment and only differed on subtle visual dimensions, using these stimuli in a repeated recognition paradigm enabled us to test whether visually-based face recognition would induce memory updating. 
We used the above face space method to manipulate similarity between distractor face images and learned target face images in order to compare the effects of active recognition attempts on face memory distortions with other control conditions that were matched in terms of the number and extent (face space distance) of recognition errors they reinforced. This matching procedure was inspired by Bridge \& Voss (2014; see also Bridge \& Paller, 2012) and constitutes an important design improvement on other prior research that has investigated how memories change as a result of active retrieval attempts but typically without controlling for how much the different tasks (e.g. retrieval vs re-study) involve attending to old/correct vs. new/erroneous information. Without such control, any differences in memory after participants complete different retrieval or study tasks could potentially be caused by participants attending more to previously encountered than new information in one task compared to another task, regardless of the other cognitive processes involved in the tasks (e.g. whether or not intentional retrieval processes are engaged).

All three experiments consisted of three main phases, repeated in two cycles. In each cycle, participants first learned a series of target faces in a study phase. In a subsequent "refresh" phase, participants were shown learned faces together with four distractors that varied in similarity (face space distance) to the target face. We manipulated the type of task participants performed on these refresh face sets across experiments and cycles to either encourage intentional recognition attempts, intentional encoding attempts, or other decision processes that were not based on recognition memory. In a final recognition test phase, the learned target faces were shown again together with the same distractors from the refresh phase, and participants were asked to recognise the face they had initially learned in the study phase. Recognition-induced updating was operationalised as the extent to which recognition errors in the refresh phase were more likely to be repeated in the final recognition test compared to other, non-repeated errors. 


\section{Experiment 1}

The first experiment investigated whether intentional face recognition attempts induced more updating than a study task that also presented previously seen faces together with distractors but where the task encouraged intentional encoding instead of recognition. We hypothesised that if recognition attempts induce face memory updating, then the final recognition test should show that participants were more likely to repeat recognition errors from a preceding active recognition task (i.e. select the same distractor across refresh and final recognition tasks) than to make non-repeated (new) errors (i.e. select a different distractor). If active recognition attempts are particularly likely to induce memory updating, then this bias towards repeating errors should be larger after an intentional recognition test than after an intentional encoding task. That is, final recognition should be more biased by recognition errors than encoding errors, even though the number and extent (face space distance) of errors were matched across the two tasks.

\section{Method}

\section{Participants}

Results are reported from 72 undergraduate participants ranging in age between 18-28, with 60 females $\left(M_{\text {age }}=19.44, S D_{\text {age }}=1.80\right)$. All participants had normal or corrected-to-normal vision and were recruited from the University of Kent. This sample size was determined by a combination of power and counterbalancing considerations as well as practical recruitment constraints, and resulted in $>.98$ power to detect a medium effect size of Cohen's $d=.5$ or $>.70$ power to detect a small effect size of Cohen's $d=.3$ at an alpha level of .05 (two-tailed). Nine additional participants were removed due to failing to comply with task instructions. 
Participants received course credits in return for participation, and the study was approved by the University of Kent School of Psychology ethics committee.

\section{Materials}

Stimuli were near photo-realistic images of artificial faces that were generated using previously established methods (Solomon et al., 2013). This approach involved constructing a mathematical representation of a multidimensional "face-space" (or "image-space"), in which unique facial images are represented by single points. The dimensions of the face space are determined by principal components that have been derived through Principal Component Analysis of real face photographs (in this case, photos of white, mostly middle-aged males and females). The 29 largest components (selected because they explained a meaningful amount of variance) were next used to create novel, artificial face images by assigning different weights to components, which is equivalent to sampling different locations in an image face space with 29 dimensions ( see Solomon et al., 2013; for further details).

Accordingly, the perceptual similarity between any two face images can be determined by varying the corresponding Euclidean distance between points in the face-space model (and indeed, control experiments showed a strong relationship between Euclidean distance and participants self-reported experience of face similarity, see Supplemental file for full details).

Using this technique, we generated 60 face "sets", that were each made up of a target face image plus four distractor face images that were randomly sampled within fairly close range of the target, to ensure images in a set shared some degree of perceptual similarity but varied in similarity within this range (see Fig. 1A for examples). The 60 different sets were sampled from maximally distant locations in face space, to minimise similarity across sets. The distance of the sets from the face space origin was kept constant to avoid differences in distinctiveness between sets. Furthermore, 30 face sets were allocated to one list (A) with 30 
other sets allocated to a second list (B), and face sets were "matched" in a pairwise way across these two lists so that all the Euclidean distances between images within sets were equal across the pairs, even though the matched sets were perceptually different from each other. As an example, if "face 1" in "set A1" was located at a distance of 1000 (arbitrary units) from "face 2" and 3000 from "face 3", while "face 2" and "face 3" were located at a distance of 2500 from each other, then the exact same pairwise distances were also used for faces A-C in "set B1", even though their actual locations in face space were different (ensuring minimal perceptual overlap between paired sets). This principle was used to ensure all distances between the five images within each set was the same across matched sets from list A and B. List A and B were assigned to either the retrieval task or the study task (assignment counterbalanced across participants), ensuring that we could match the amount of error introduced during refresh across the retrieval versus the study blocks, because participant errors in the retrieval task determined which faces they would be asked to encode in the subsequent study task (see Procedure).

The generated face images varied in terms of face shape and colour, internal facial features and the relationships between features, while external features such as hair and ears were averaged so that the resulting images consisted of a clear face with a blurry darker outline (Fig. 1A). Thus, the faces varied only on perceptual characteristics, and whilst participants could potentially extract a few semantic details such as the likely gender or age from the images, these factors were not systematically nor categorically manipulated (for example, faces varied in terms of how "masculine" vs. "feminine" they looked since the principal components that related to variance in gender could have different values). Also, any such semantic details would not help participants recognise targets among their set of similar distractors. Therefore, recognition of face images in this task was likely based on visual characteristics, with minimal influence from semantic memory. 


\section{Figure 1}

Illustration of Experiment 1-3 stimuli, procedure, and the final recognition bias measure.

A

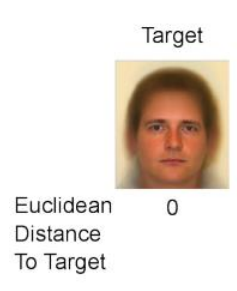

B

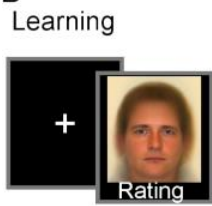

Refresh (Recognition / Study / Distinctiveness Task)

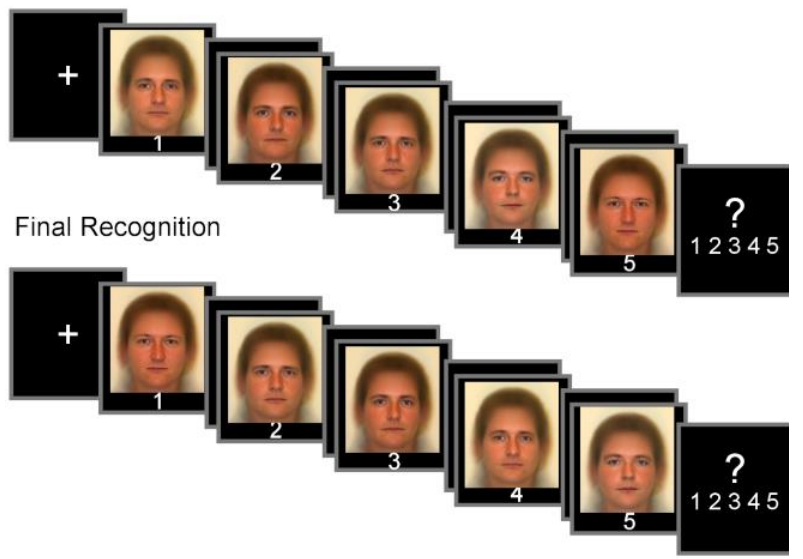

C

Final Recognition Bias Measure

Distractor Selected During Refresh

- Biased Error

- . . Non-biased Error

- Correct Target Selection (not included in recognition bias analysis)

Final Recognition Test Response Alternatives
Face Similarity Manipulation

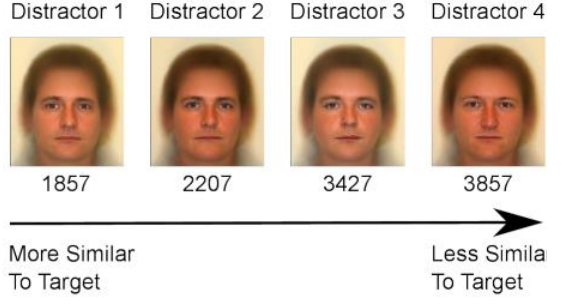

Trial Structure
?

345

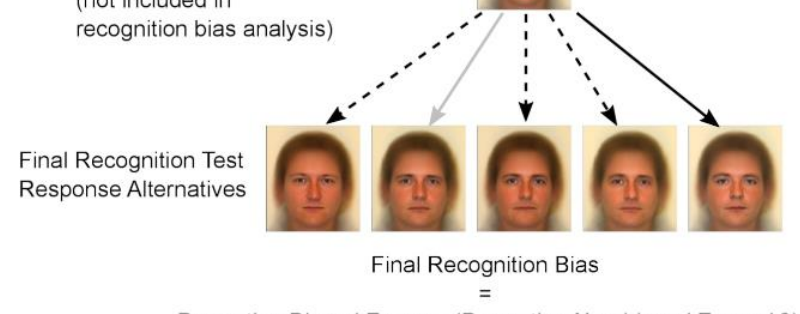

Proportion Biased Errors - (Proportion Non-biased Errors / 3)

Note. A) Example stimuli showing the manipulation of face similarity. B) Procedure in the three experiments. Participants first learned face targets. In a following refresh phase, learned faces were shown amongst four distractor faces and we manipulated the refresh task across blocks and experiments. The task required either intentional recognition judgements (recognition), intentional encoding attempts (study), or judgements that were not based on recognition memory (selfchoice). In the final recognition phase, participants were asked to indicate which of the five faces they recognised from the initial learning phase. C) Illustration of the final recognition bias measure. Final recognition trials where a distractor had previously been selected in the refresh phase were sorted into biased error trials (the same distractor was selected in the final recognition test, solid line), nonbiased error trials (a different distractor was selected in the final recognition test, dashed line) and correct target selection (grey line). Next a final recognition bias measure was calculated by subtracting each participant's probability of making a non-biased error from their probability of making a biased error, with higher scores indicating more bias towards previous errors. 
The experiment was completed on a Dell Optiplex 9020 desktop computer with PsychoPy (Peirce, 2009) and responses were input by keyboard presses. The screen measured $51 \mathrm{~cm} \times 28.4 \mathrm{~cm}$, with a resolution of $1920 \times 1080$ pixels. The face images subtended a $5.12 \times$ 5.88 visual angle when participants sat at a distance of $75 \mathrm{~cm}$ from the screen in all tasks. All participants completed the experiment on the same computer in a Psychology laboratory cubicle.

\section{Procedure}

Participants completed two blocks, which each contained an initial face learning phase, which was followed by a "refresh" phase and a final recognition phase (Fig. 1B). The only difference between blocks was the type of task completed during refresh and the face stimuli shown (list A or B, counter-balanced across participants). In block one refresh, participants completed an 'active' recognition task requiring retrieval attempts, whereas in block two refresh, they completed an intentional encoding task that therefore did not involve active retrieval instructions (a "passive" task). A filler task was used to clear visual working memory after the learning and refresh phases within each block, and participants received a short break in between blocks.

Learning. In each block, participants first completed a face learning task where all 30 face targets were shown in three cycles. For each cycle, face targets were presented one at a time for $5000 \mathrm{~ms}$ each in a randomised order, preceded by a 500ms fixation cross, and participants were instructed to attempt to memorise these face images for a later recognition test while also rating each face target on different aspects in order to facilitate encoding. During cycle one, participants decided whether the face depicted a person that appeared trustworthy (key press 5) or not trustworthy (key press 1). Cycle two involved rating faces as attractive (key press 5) or unattractive (key press 1). Finally, the third cycle required 
participants to indicate whether a face was feminine (key press 5) or masculine (key press 1). These ratings were informed by Oosterhof \& Todorov (2008) who showed that evaluation of these different traits tend to be based on different dimensions of face images. Participants received instructions for each rating task prior to beginning each cycle and made their responses whilst the face was on screen. A validation study with a different sample $(N=22)$ showed that this learning procedure led to sufficient encoding since participants could accurately discriminate target faces from completely new faces if given a standard old/new recognition task (hit rate: $M=.72, S E=.02$; correct rejection rate: $M=.65, S E=.03 ; \operatorname{Pr}: \mathrm{M}=$ $.38, \mathrm{SE}=.03$; see Supplemental file for full details).

Recognition during refresh (block one). In block one, participants were given active recognition instructions during the refresh phase. They were shown 20 of the 30 faces that had just been encoded in the prior learning phase. For each trial, a target face was presented together with the four distractor faces from the corresponding face set. These five faces were presented sequentially to ensure participants focused on all faces in a trial for an equal duration. Face images were shown at the centre of the screen for $1250 \mathrm{~ms}$ each, with a number below (numbered 1-5 in the order they appeared) and preceded by a $1000 \mathrm{~ms}$ fixation cross. Across the 20 trials, the face target image was located in each of the five positions an equal number of times, but the presentation order of face sets across trials was randomised. After viewing all five faces, a question mark was shown on the screen to cue participants to press 1-5 on the keyboard to indicate the position of the face they recognised to be the same face they had seen during learning. Importantly, participants were told to pay full attention throughout and consider all five faces before making their decision as to which face was the target during the response screen. After responding, the next recognition trial began.

Study during refresh (block two). In block two, participants were given an intentional encoding task during the refresh phase. Face sets used in the second block were 
the matched sets taken from the list that was not shown during block one (i.e. if list A was used in block one, list B was used in block two). The study task used a near identical trial format as the refresh recognition task, with the five faces from a set presented sequentially, numbered from 1-5 and shown for $1250 \mathrm{~ms}$ each, with a $1000 \mathrm{~ms}$ fixation cross preceding each face. Apart from instructions, the only difference between this study refresh task and the recognition refresh task was that four of the five fixation crosses that were presented before face images were coloured grey, with one fixation cross presented in white. The white fixation cross was used as a cue for participants to selectively encode the face that immediately followed the white cross. Participants were instructed to pay attention to all faces shown but try their best to learn the cued face because they would be asked to recognise it in a subsequent recognition test (although these instructions were deceptive, unbeknown to participants). After seeing all five faces, participants pressed the key associated with position of the face they had been asked to encode (1-5).

Importantly, the faces that participants were asked to intentionally encode during study refresh were determined by participants' responses during the recognition task refresh phase in block one. That is, if participants thought they recognised the first image of a particular set (e.g. "set A1") as the target during the refresh recognition task, then the first image would be preceded by a white fixation cross for the corresponding matched set (e.g. "set B1") during the study refresh task. Critically, the faces matched by position were also matched according to face space distances within a set, so if participants selected the correct target face for a particular recognition test set, they were also asked to encode the correct target face for the matched set in study. If they made an error and selected a distractor face for a particular recognition test set, then they were also asked to encode a distractor for the matched study set, and the similarity (face space distance) between the distractor and the target face was equal across the two matched sets. This procedure was followed for all refresh 
trials in order to ensure that the faces participants were asked to encode during study were equal in error to faces selected in the refresh recognition task. Because of this design feature, the study task was always completed in block two (similar to Bridge \& Voss, 2014). The presentation order of sets was randomised within each task, thus there was no systematic order relationship between the two refresh tasks.

Final recognition. The final recognition task in both blocks involved the same trial format as described in the refresh recognition task, but with all 30 sets presented in a random order. Of these, 20 'repeated 'sets had been shown during the previous refresh task, and 10 'baseline' sets had not been shown during refresh. For repeated trials, the order of the five faces within each trial was randomly reshuffled from the previous refresh phase. Importantly, participants were instructed to focus on recognising the face they had seen during the learning phase, and not base responses on what faces that they had selected or been asked to encode in the previous refresh task (that is, they were asked to disregard the refresh task completely). Baseline trials were included to test for potential effects of refresh tasks on recognition accuracy, but since those results are not central to the updating hypotheses they are presented in a supplementary analysis (see Supplementary file). Briefly, we found no difference in final recognition accuracy between repeated and baseline sets after any refresh tasks in any experiment.

Letter search filler task. Following all learning and refresh phases, participants completed a visual letter search (Treisman \& Gelade, 1980) filler task to clear visual working memory. Seventy-two letter arrays, containing a mixture of letter type $(X, O \& N)$, frequency $(1,5,15 \& 30)$ and colour (red, green \& blue), were presented one at a time. Participants were tasked with searching for a blue letter which was present in $18 / 72$ pictures. Participants indicated whether the array had a blue letter (key press 5) or did not have a blue letter (key press 1). Trials were self-paced, with all 72 trials completed in around 1 minute. 


\section{Data Analysis}

Data were extracted with MATLAB (version 17A; MathWorks, Inc, Natick, MA) and analysed with JASP. A final recognition bias measure was calculated to determine the extent to which participants were biased by prior refresh errors during final recognition, in order to assess the influence of recognition attempts versus intentional encoding on face memory updating (Fig. 1C). This analysis included only final recognition repeated trials where a distractor face had been previously selected during the initial refresh phase (i.e. falsely recognised as a target by the participant during the refresh recognition block or indicated for encoding during the refresh study). For those "prior error" repeated trials, the proportion of trials where the same distractor face was selected during final recognition was calculated. Next, the proportion of "prior error" trials where participants selected a different distractor face during final recognition was calculated, and was divided by three in order to calculate the probability of participants making a non-biased error on the final test. To provide a single measure of final recognition bias, the probability of non-biased error responses was subtracted from the probability of biased error responses to provide a final recognition bias difference score. This measure therefore excluded trials where participants selected the target face in either the refresh task or final test, so that comparisons across tasks were not confounded by differences in how often participants selected the target face. A positive bias difference score indicates that participants were biased towards recognising distractor faces on the final test that they had previously either selected during refresh recognition attempts or been asked to encode during refresh study, whereas a negative bias score would indicate a bias away from those distractor faces. Hence, it was predicted that the bias difference score should be significantly larger than zero if participants' face memories had become updated by recognition/study during the refresh phase. If face memory updating is enhanced by active 
recognition attempts, then this bias score should be more positive following recognition compared to study refresh.

In addition to the key analysis of bias described above, the proportion of trials where the target face was selected was also measured to assess participants' ability to correctly recognise target faces during refresh and final recognition. Since these analyses were not of key theoretical interest, only descriptive results are presented here to aid interpretation of the bias results (see Supplemental file for statistical analysis of target selection rates across refresh, final recognition repeated and baseline trials).

Null-hypothesis significance testing with standard GLM tests ( $t$-tests and ANOVAs) was used to infer meaningful differences in final recognition bias between the refresh recognition and study tasks. We also calculated Bayes Factors for all $t$-tests to compare the ratio of evidence in support of the alternative hypothesis of a difference in means in either direction, versus the null hypothesis of no difference in means (see Dienes, 2011). Bayes Factors for the alternative over the null hypothesis $\left(\mathrm{BF}_{10}\right)$ can be interpreted as relative evidence in favour of the alternative (values greater than 3), in favour of the null (values lower than 0.33 ), or not clearly supporting either hypothesis (values close to 1). All Bayes factors were calculated with two-tailed tests, using JASP (JASP Team, 2019) with recommended default priors (a Cauchy distribution with centre $=0, r=0.707$ ).

\section{Results}

\section{Target selection rates}

Due to the matching procedure, the proportion of targets selected during refresh was equivalent during recognition and study tasks, and showed that participants selected target 
faces during refresh recognition for half of the trials on average $(M=0.50 ; S D=0.17)$. Target selection rates (i.e. accurate recognition responses) during final recognition for repeated trials was $0.45(\mathrm{SD}=0.17)$ in block one and $0.39(\mathrm{SD}=0.16)$ in block two.

\section{Final recognition bias}

Final recognition bias (Fig. 2) was significantly higher than 0 following both the recognition refresh task, $M=.14,95 \% \mathrm{CI}[.09, .18], t(71)=6.16, p<.001, d=0.73, \mathrm{BF}_{10}=324550.83$, and following the study refresh task, $M=.06,95 \% \mathrm{CI}[.02, .10], t(71)=3.17, p=.002, d=$ $0.37, \mathrm{BF}_{10}=12.04$, indicating that participants were more likely to erroneously recognise previously selected distractor faces as the target than to recognise previously unselected distractor faces as the target after both refresh tasks.

These results therefore indicate that making errors during active recognition attempts and intentionally encoding errors during study both updated face memories, presumably caused by encoding of selected distractor faces during refresh in both tasks. However, the final recognition bias difference score was significantly higher following refresh recognition than study, $t(71)=2.80, p=.007, d=0.33, \mathrm{BF}_{10}=4.73$, consistent with the prediction that active recognition attempts enhance memory updating compared to intentional encoding. 


\section{Figure 2}

Recognition bias during the final recognition test following different refresh tasks across the three experiments.

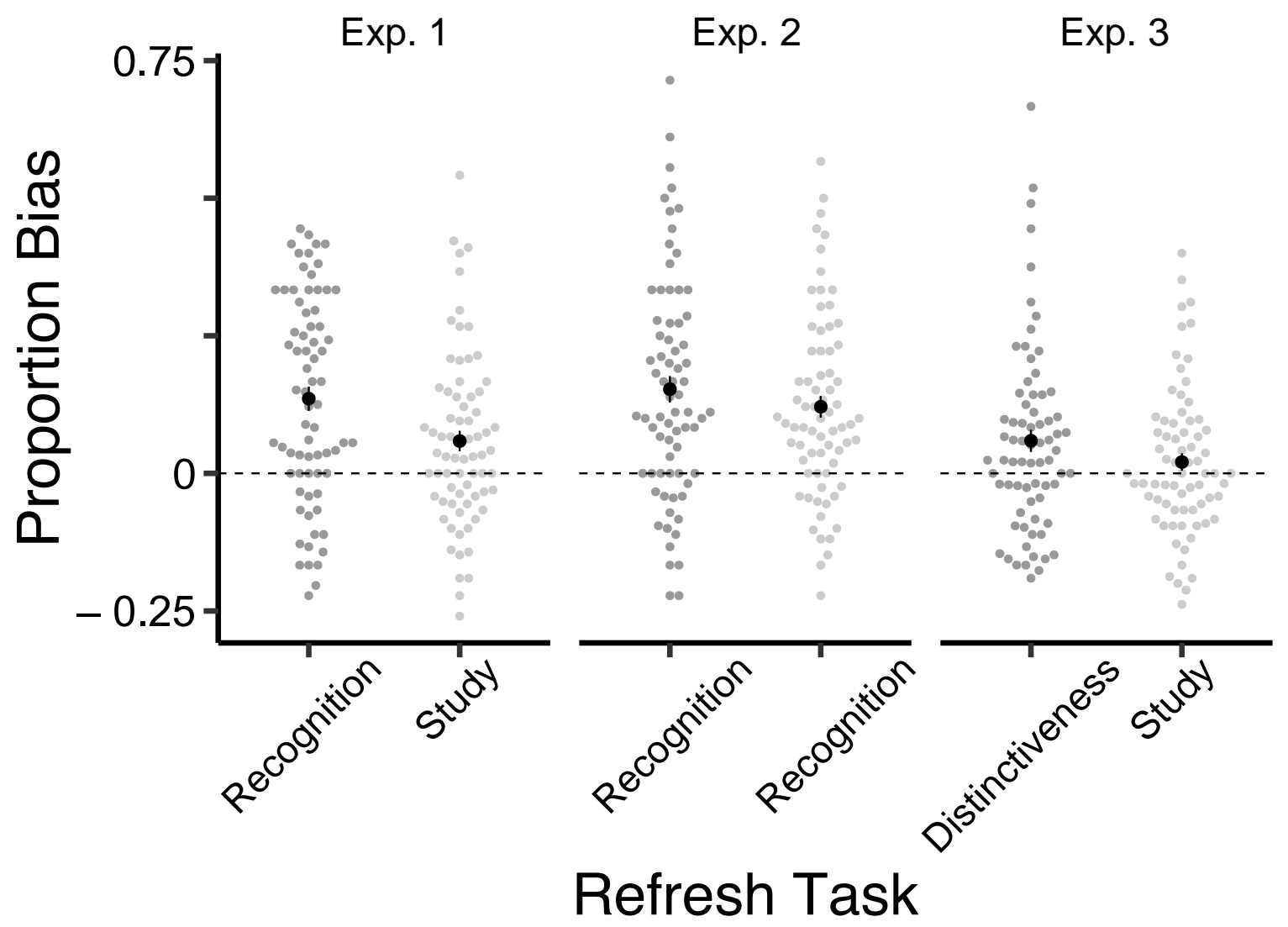

Note. Black circles show the sample mean bias score for each condition, with standard errors of those means indicated by lines. Grey circles show individual participants' bias scores.

\section{Discussion}

Experiment 1 showed that participants' face memories during final recognition were more strongly biased towards previously wrongly selected distractor faces when those faces had been chosen during active recognition attempts than when participants had been instructed to encode them. These results therefore support the view that active retrieval processing is a 
stronger inducer of memory updating compared to studying the same materials, consistent with findings that testing compared to re-study is a particularly potent method for strengthening semantically meaningful memories (e.g. Gao et al., 2016; Karpicke, 2017; Roediger \& Butler, 2011). In line with Bridge and Voss (2014), we ensured that selected responses in the study condition introduced the same amount of error as participants' responses in the recognition refresh task, both in terms of number of errors as well as the similarity of errors to the correct response. Therefore, the observed increase in face recognition bias following recognition attempts compared to encoding attempts is more likely due to differential engagement of active retrieval processes than confounding differences in the extent to which the two tasks reinforced previously learned information.

However, one important caveat is that the results from Experiment 1 could be subject to order effects, given that the recognition condition always preceded the study condition, which was necessitated by the matching procedure. This means that confounds such as fatigue could have influenced memory performance, which could have reduced final recognition bias in the second study block compared to the first recognition block. To address this issue, a second experiment was conducted to test whether the results from Experiment 1 could be explained by order effects.

\section{Experiment 2}

Experiment 2 had an almost identical design to Experiment 1, where participants completed two blocks of learning, refresh, and final recognition tasks (separated by filler tasks as in Experiment 1). The only difference for Experiment 2 was that participants completed an active recognition task in the refresh phase in both blocks, thus keeping the task constant and enabling us to assess the effect of order on final recognition bias. If the 
increased bias following recognition compared to study in the first experiment was caused by order effects, then final recognition bias should be larger in block one compared to block two also in Experiment 2, even though both blocks now involved the same recognition task during refresh. In contrast, if active recognition processes enhance memory updating, then final recognition bias should be similar across blocks one and two.

\section{Method}

\section{Participants}

Seventy-two undergraduate participants with an age range of 18-28 (62 female, $M_{\text {age }}=19.29$, $\left.S D_{a g e}=1.52\right)$ were recruited from the University of Kent, with sample size decided a-priori to be kept constant with Experiment 1. All participants had normal/corrected to normal vision, were naïve to the experiment aims, and had not participated in any previous studies with similar stimuli or tasks. Participants received course credits in return for participation, and the study was approved by the University of Kent School of Psychology ethics committee.

\section{Materials, Procedure and Data Analysis}

All materials and procedure for Experiment 2 was identical to Experiment 1 with the exception that participants completed a recognition test during both refresh phases. This refresh recognition task was identical to the task conducted in refresh block one in Experiment 1. As in Experiment 1, assignment of face set list A and B to block order was counterbalanced across participants.

The same final recognition bias difference score as used in Experiment 1 was calculated separately for blocks one and two, and compared across blocks using the same statistical approach as in Experiment 1. 


\section{Results}

\section{Target selection rates}

The mean target selection rates (i.e. accurate recognition responses) during refresh was 0.55 $(\mathrm{SD}=0.16)$ during block one and $0.46(\mathrm{SD}=0.17)$ during block two indicating that, similar to Experiment 1, participants were on average selecting distractor faces during refresh recognition for approximately half of the trials. The mean final recognition target selection rate for repeated trials was $0.49(\mathrm{SD}=0.16)$ in block one and $0.42(\mathrm{SD}=0.17)$ in block two.

\section{Final recognition bias}

Mean final recognition bias was significantly higher than 0 in blocks one, $M=.15,95 \% \mathrm{CI}$ $\left.[.11, .20], t(71)=6.35, p<.001, d=0.75, \mathrm{BF}_{10}=693988.96\right)$ and two, $M=.12,95 \%$ CI $[.08$, $\left..16], t(71)=6.14, p<.001, d=0.72, \mathrm{BF}_{10}=301108.83\right)$, replicating the results from Experiment 1 that participants were more likely to repeat erroneous recognition responses from refresh in the final recognition test than switch between distractor recognition responses across the two tests. This finding therefore suggests that both refresh recognition blocks resulted in memory updating/encoding of face distractors during refresh. However, there was no significant difference in final recognition bias between block one and two, $t(71)=1.06, p$ $=.292, d=0.13$. Furthermore, the Bayes Factor for this contrast showed that the evidence favoured the null over the alternative hypothesis $\left(\mathrm{BF}_{10}=0.22\right)$ suggesting that both recognition refresh tasks induced an equivalent degree of face memory updating. 


\section{Discussion}

Experiment 2 was conducted to establish whether the evidence for enhanced recognitioninduced updating in Experiment 1 was caused by differences in cognitive processing engaged by the manipulated refresh tasks, or could be explained by an order confound since the recognition block was always conducted before the study block. In Experiment 2, participants therefore completed exactly the same tasks in two blocks, meaning the only systematic difference between blocks was the order in which they appeared (although the stimuli were different across blocks, assignment of stimuli to block was counterbalanced across participants). We then tested if face memory updating was susceptible to order effects, which would be expected if there was a drop in final recognition bias from block one to two. The results from Experiment 2 however showed that recognition bias was highly significantly larger than zero in both blocks and not significantly different across blocks, and the Bayes Factors provided evidence in support of no difference in updating between the two blocks. The results thus show that updating of face memories was not susceptible to order effects in the current experimental design. Collectively, the data from Experiments 1 and 2 therefore suggests that active recognition attempts are particularly likely to induce face memory updating when compared to intentional encoding attempts.

However, just because a recognition test induces more memory updating than a restudy task does not mean these updating differences are necessarily caused by different engagement of core retrieval processes across the tasks. Recognition and re-study tasks also differ in other regards, such as participants' ability to choose their response. Previous research on the "self-choice" effect show that participants typically have better memory for stimuli they have chosen to encode during a learning phase, compared to stimuli that were chosen by the experimenter for participants to learn (e.g. Coverdale \& Nairne, 2019; Watanabe \& Soraci, 2004). Furthermore, people's memory is enhanced by the opportunity to 
choose aspects of their learning experience even when those choices are irrelevant for later tested information, and is associated with enhanced hippocampal-striatal interactions during encoding (Murty et al., 2015) suggesting that the act of choosing enhances subsequent memory by engaging memory-related brain networks. It has been proposed (e.g. Bridge \& Paller, 2012) that retrieval-induced updating occur when encoding mechanisms are engaged during retrieval attempts, causing people to subsequently remember information from the retrieval event rather than information that was encoded during the original event. However, it is possible that encoding mechanisms may be engaged in retrieval tasks because such tasks involve actively making choices, rather than because of retrieval-specific processes such as reactivation of a stored memory trace. In the current experiments, the active recognition task could have enhanced face memory updating because it involved choosing one of the five faces, in contrast to the study task where participants were instructed to encode one of the five faces.

The self-choice effect on memory has primarily been examined in the context of initial encoding of novel stimuli (Coverdale \& Nairne, 2019; Murty et al., 2015; Watanabe \& Soraci, 2004), rather than as a post-learning mechanism for memory updating. However, research on the "testing effect" suggests that active retrieval attempts ("retrieval mode") enhances memory for previously learned material more than semantic generation tasks where the participant is not actively trying to recall the encoding context, but are still generating and choosing a response (e.g. Karpicke \& Zaromb, 2010). This line of research therefore suggests that intentionally attempting retrieval is a particularly potent factor for memory updating over-and-above any effects of self-choice on updating. Prior studies in this domain have however focused on how memories for original experiences are enhanced by testing, and it is not clear therefore whether errors made during recognition attempts leads to more memory 
distortions compared to when people choose "erroneous" responses in a non-memory task. This issue was investigated in the next experiment.

\section{Experiment 3}

Experiment 3 was conducted to assess whether the evidence for recognition-induced updating in Experiments 1 and 2 could be explained by enhanced encoding of self-chosen faces, regardless of whether the basis for that choice was memory retrieval. As in previous experiments, participants completed two blocks of learning, refresh and final recognition tasks (separated by filler tasks). We compared a self-choice refresh task during block one with a study (intentional encoding) refresh task in block two, while keeping all other aspects of the design identical to the previous experiments. In the self-choice task, participants were asked to indicate which of the shown five faces they thought was the most distinctive. Crucially, this task therefore maintained participants' ability to select any of the five faces for their response whilst removing the need to use recognition judgements to guide their decision. The self-choice task was conducted prior to the intentional encoding task so that we could match the extent to which the two tasks required participants to focus on previously seen versus new face information. That is, as in Experiment 1, participants responses on the self-choice task determined what faces they would be asked to encode in the study task, so that we could ensure that participants' memory for targets was reinforced to the same extent on both tasks, and that the number of errors and the face space distance of those errors that were selected across tasks were equal. We then tested whether these different refresh tasks induced different degrees of bias on a final recognition test. If the act of choosing enhances memory updating, then final recognition bias should be larger following the self-choice task than the study task. If there are no differences in final recognition bias following self-choice 
and study refresh tasks however, that finding would suggest that the cognitive processes associated with active recognition attempts specifically enhanced memory updating in the previous experiments.

\section{Method}

\section{Participants}

Seventy-two undergraduate participants ranging in age between 18-28 (59 female, $M_{\text {age }}=$ $\left.19.86, S D_{\text {age }}=1.46\right)$ were recruited from the University of Kent, with sample size decided apriori to be kept constant with Experiments 1 and 2. All participants had normal/corrected to normal vision, were naïve to the experiment aims, and had not participated in any previous studies with the same stimuli, including Experiments 1 and 2. Participants received course credits in return for participation, and the study was approved by the University of Kent School of Psychology ethics committee.

\section{Materials, Procedure, and Data Analysis}

All materials and procedure for Experiment 3 was identical to Experiment 1 with the exception that participants completed a distinctiveness judgment task during the first refresh phase. In this task, participants were instructed to select the face they thought was most distinctive amongst the one target and four distractor faces shown per trial. Crucially, participants were not told that each trial included target and distractor faces (unlike in the recognition refresh tasks where participants were told that a previously seen target was intermixed with distractors in each trial). Furthermore, no explicit instructions were given to participants to try to recognise faces from the learning phase, meaning that the task was unlikely to encourage intentional retrieval attempts. Participants completed an intentional 
encoding task during the second refresh phase, which was identical to the study task in Experiment 1. As in Experiment 1, the faces that participants were asked to encode during this study refresh task were based on responses they had previously given to matched sets during the self-choice task, ensuring that we could equate the amount of error introduced during refresh across the distinctiveness versus the study blocks. As in the first two experiments, assignment of face stimuli set list A and B to block order was counterbalanced across participants.

The same final recognition bias difference score as used in Experiments 1 and 2 was calculated separately for blocks one and two, and compared across blocks using the same statistical approach as previously.

\section{Results}

\section{Target selection rates}

The average target selection rates during the distinctiveness refresh task was $0.32(\mathrm{SD}=$ 0.17). Since target selection rates in the self-choice task were substantially lower than in the active recognition tasks in Experiments 1 and 2, this suggests that participants were following instructions and not relying on active recognition attempts when making distinctiveness judgements. Because responses in the self-choice task determined which faces participants were asked to encode in the study refresh task, participants were also asked to encode targets on the same proportion of trials in the study task. Target selection rates (i.e. accurate recognition responses) during final recognition for repeated trials was $0.47(\mathrm{SD}=0.15)$ in block one and $0.39(\mathrm{SD}=0.14)$ in block two. 


\section{Final recognition bias}

The final recognition bias difference score was significantly higher than 0 following the selfchoice refresh task, $M=.06,95 \%$ CI $[.02, .10], t(71)=2.93, p=.005, d=0.35, \mathrm{BF}_{10}=6.48$, but was not significantly different to 0 following the study refresh task, $M=.02,95 \%$ CI [$\left..01, .05], t(71)=1.30, p=.199, d=0.15, \mathrm{BF}_{10}=0.29\right)$. These results indicate that participants were biased towards erroneously recognising distractor faces as shown during initial learning if they had chosen those faces as most distinctive in the first refresh phase, since they were more likely to select those distractors than other distractor faces on the final recognition test. However, after study, participants were just as likely to select any of the four distractor faces during final recognition.

The final recognition bias difference measure was however not significantly different when comparing blocks one and two directly $(t(71)=1.53, p=.132, d=0.18)$. The Bayes Factor for this contrast provided anecdotal evidence relatively more in favour of no difference than a difference in bias between the blocks $\left(\mathrm{BF}_{10}=0.39\right)$, however it should be noted that the $\mathrm{BF}_{10}$ was not smaller than 0.33 , which is the threshold typically considered "substantial" evidence in favour of the null hypothesis. Thus, these findings provide tentative, but not conclusive, evidence that face memory updating is not enhanced due to the act of choosing when compared with intentional encoding during study.

\section{Discussion}

In Experiment 3, we tested whether face memory updating was enhanced following a distinctiveness judgement task that involved freely choosing between different alternative responses but did not require active face recognition attempts. The results showed that final recognition responses were somewhat biased towards the previously chosen distractor faces 
(because our estimate of bias was larger than zero), but there was no evidence for a significantly enhanced updating effect of this distinctiveness judgement task when compared to the effects of an intentional encoding task. However, the latter study task did not induce significant updating in this experiment, in contrast to the significant bias induced by intentional encoding in Experiment 1.

Taken together with Experiments 1 and 2, which showed evidence for enhanced subsequent bias following a recognition task, the results therefore suggests that the cognitive processes engaged during active retrieval attempts are particularly potent for inducing updating of face memories, consistent with prior research (Bridge \& Voss, 2014; Karpicke \& Zaromb, 2010). Therefore, although prior research has shown that self-choice enhances encoding of novel information (Murty et al., 2015; Watanabe \& Soraci, 2004) it may not enhance updating of memories that have already been encoded to the same extend as active retrieval attempts do. However, in order to draw this conclusion, the updating effects of active recognition attempts need to be directly compared with updating effects of the selfchoice task, as addressed in the next, between-experiment analyses.

\section{Between experiment analyses}

Two additional analyses assessed the effects of different refresh manipulations on final recognition bias directly across experiments while holding order constant. In these analyses, we used independent t-tests coupled with Bayes Factors (using default priors, as in previous analyses) to test the relative evidence for a between-experiment difference $\left(\mathrm{H}_{1}\right)$ or no difference $\left(\mathrm{H}_{0}\right)$ in recognition bias within blocks one and two separately. 


\section{Recognition bias difference between Experiments 1 and 2}

The first analysis compared Experiments 1 and 2 to test the recognition-induced updating hypothesis against an order effect hypothesis directly. If order effects account for the patterns of bias during final recognition, then no differences should be found between Experiments 1 and 2 in either the first or second blocks, as the amount of bias should be similar across blocks in both experiments. However, if face memory updating is enhanced by cognitive processes engaged during recognition as opposed to study, then recognition bias should be significantly larger in Experiment 2 than in Experiment 1 for the second block only, since the second block of Experiment 2 involved a recognition refresh task but the second block of Experiment 1 involved a study refresh task.

Planned comparisons (Table 1) showed that final recognition bias was not different across Experiments 1 and 2 during the first block and the Bayes Factor indicated support for no difference in Block 1 bias between the two experiments, as would be expected. As predicted, final recognition bias in the second block was however significantly larger in Experiment 2 (i.e. following an intentional recognition test) than Experiment 1 (i.e. following an intentional encoding task), and the Bayes Factor for this comparison provided anecdotal evidence in support of a difference. Therefore, taken together these results support the argument that recognition attempts are more likely than intentional encoding to induce face memory updating. 


\section{Table 1.}

Between-experiment differences in recognition bias.

\begin{tabular}{lllllllll}
\hline & \multicolumn{4}{l}{ Experiment 1 vs. 2 } & \multicolumn{6}{c}{ Experiment 1 vs. 3 } \\
\hline Comparison & $t$ & $p$ & $d$ & $B F_{10}$ & $t$ & $p$ & $d$ & $B F_{10}$ \\
Block 1 recognition bias & 0.53 & .597 & 0.09 & 0.20 & 2.56 & .012 & 0.43 & 3.46 \\
Block 2 recognition bias & 2.30 & .023 & 0.38 & 1.97 & 1.57 & .119 & 0.26 & 0.55 \\
\hline
\end{tabular}

Note. Bayes Factors $\left(\mathrm{BF}_{10}\right)$ represent relative evidence in favour of a difference $(>3)$, no difference $(<0.33)$ or are inconclusive $(\sim 1)$. Degrees of freedoms were 142 for all acrossexperiment analyses.

\section{Recognition bias difference between Experiments 1 and 3}

The second analysis compared recognition bias between Experiments 1 and 3 in order to test a recognition-induced updating hypothesis against a self-choice effect hypothesis directly. If recognition attempts enhance the updating of face memories, then final recognition bias in the first block of Experiment 1 should be larger than final recognition bias in the first block of Experiment 3, since a recognition refresh task was conducted in Experiment 1 and a selfchoice refresh task was conducted in Experiment 3 during the first blocks. In contrast, if actively choosing a face was the critical factor that enhanced updating regardless of whether this choice was based on recognition judgements, then there should be no difference in final recognition bias between Experiment 1 and Experiment 3. Either way, no difference was expected between experiments for recognition bias in block two, since both Experiments involved a study task during the refresh phase in block two.

Planned contrasts (Table 1) showed that final recognition bias was significantly larger in block one for Experiment 1 (i.e. following an active recognition test) compared to Experiment 3 (i.e. following a self-choice task without intentional retrieval requirements), and this difference was also supported by the Bayes Factor. There was no difference between experiments in final recognition bias for block two trials as was expected (although the Bayes 
Factor for this contrast was inconclusive). These results suggest that the enhanced face memory updating seen after active recognition attempts is not fully explained by the recognition task involving an element of choice. Instead, the findings are more consistent with the notion that trying to intentionally retrieve memories is particularly likely to induce the updating of face memories.

\section{General Discussion}

In three experiments, we investigated whether active recognition attempts induce updating of face memories. Prior research has shown that recalling meaningful and complex event memories can induce memory distortions because errors made during retrieval attempts become encoded and bias subsequent remembering (e.g. Bridge \& Paller, 2012; Marsh et al., 2007), but it was not clear based on previous literature whether visually-based recognition of faces would induce similar distortions. The first experiment showed that face recognition attempts did induce updating, and did so to a greater extent than an intentional encoding task that also exposed participants to previously encountered faces but did not require long-term memory retrieval. Experiments two and three were conducted to determine the role of retrieval-specific processes in this finding. In experiment two, we established that active recognition attempts enhanced updating regardless of the order in which the tasks were conducted. In experiment three, we established that active recognition attempts resulted in larger face memory distortions than a control task where decisions about previously seen faces were not based on recognition memory. This finding thus suggests that recognitioninduced updating of face memories was not solely caused by general decision-related processes (Murty et al., 2015; Watanabe \& Soraci, 2004) but instead was particularly enhanced by the engagement of intentional retrieval processes (Karpicke \& Zaromb, 2010). 
In sum, the results suggest that recognition attempts can induce updating of face memories, similarly to how recall updates richer and more complex event memories.

Our design was inspired by recent research showing that active retrieval of objectlocation memories enhanced updating compared to passive exposure to object-location associations that were matched in terms of how much they deviated from a previous memory (Bridge \& Voss, 2014). We applied this principle to face recognition by ensuring that the number of selected distractors and their face space distance from target faces were matched across tasks, thereby achieving a high degree of control over the extent of "error" introduced by tasks. That is, the faces that participants selected with their behavioural response, and presumably therefore attended to the most, deviated to the same extent from previously seen target faces across matched tasks. Therefore, our tasks did not differ in how much the reexposed participants to previously learned vs. new information, thereby avoiding a confound that has affected much prior research on the effects of retrieval on memory (see e.g. discussion in Karpicke, 2017). Differences in final recognition performance after different refresh tasks were therefore likely caused by differences in the cognitive processes engaged by task instructions rather than the extent to which the tasks reinforced previously learned information vs. introduced novel information.

We also ruled out general decision factors associated with actively choosing a response (e.g. Murty et al., 2015; Watanabe \& Soraci, 2004) as being the primary source of subsequent recognition biases. Asking participants to select the most distinctive faces without relying on memory did result in some subsequent bias towards selected distractor faces, but it did not lead to as large biases as the active recognition task. We chose this judgement as a control task because distinctiveness has been found to enhance face encoding in other contexts (e.g. Winograd, 1981). Thus, the effects of active retrieval attempts on face memory 
updating was larger than two control tasks that were chosen to be particularly likely to enhance distractor encoding - an intentional encoding task and a distinctiveness task, suggesting that recognition is indeed a particularly potent route to memory updating.

The face recognition test we used to induce updating required very fine-grained discrimination between perceptually similar face stimuli. Because this task was relatively challenging, it may have resulted in "desirable difficulty" during retrieval (Bjork et al., 2015), which has been found to strengthen successfully retrieved memories in the "testing effect" literature. In this line of research, it has been found that recognition tests can induce memory strengthening (Rowland, 2014), including when recognition is tested in multiple-choice format (Little et al., 2012). Furthermore, selecting incorrect alternatives in multiple choice tests can result in encoding and subsequent misremembering of those incorrect answers as being correct (the "negative testing effect", Marsh et al., 2007; Roediger III \& Marsh, 2005), This literature has also found that intentional retrieval attempts ("retrieval mode", Karpicke \& Zaromb, 2010) are particularly potent at strengthening memories compared to when previously seen stimuli are encountered without intentional attempts to remember a past event. Although re-exposure to previously seen stimuli can elicit retrieval automatically without people being in retrieval mode (e.g. Berntsen, 1996; Richardson-klavehn et al., 1994), intentional retrieval attempts likely lead to greater reactivation of stored memories compared to when previously seen stimuli are encountered in a non-retrieval processing state (Rugg \& Wilding, 2000; Tulving, 1983), thereby making intentionally retrieved memories more susceptible to changes. The testing effect literature has however typically focused on semantically rich materials in educational settings, whereas our results show distortions after erroneous recognition of distractor faces based on their visual characteristics. Therefore, updating of face memories is unlikely to be caused by elaborative retrieval of sematic 
associations, which has been suggested as a key factor in testing effects for meaningful materials (Carpenter \& Kelly, 2012; see also Ferreira \& Wimber, 2021).

The finding that visually-based face recognition can induce updating has theoretical implications for current models of memory updating that emphasise the reconstructive nature of recollection as a source of memory distortions (e.g. Schacter et al., 2011). Recollection is necessary when receiving a partial cue to a stored memory and associations between different items and/or their spatiotemporal context need to be reconstructed, but recollection may not always be needed when recognizing previously encountered items, since item recognition may rely on more automatic assessments of item "strength" in memory that give rise to a feeling of familiarity (e.g. Horner et al., 2015; Ranganath \& Ritchey, 2012; Yonelinas, 2002). Furthermore, recognition of faces based on their visual characteristics (i.e. previously unfamiliar faces encoded without additional semantic information) is thought to be particularly independent of hippocampally-mediated recollection (Bird, 2017). These theoretical accounts suggest that visually-based face recognition should not necessarily induce memory updating, in contrast to our findings.

If our findings were driven by reactivation-induced updating of stored face memories as we suggest, then why would distractor faces elicit such reactivation and subsequent biases? One possibility is that mistakenly recognized distractors acted as partial cues that reactivated the original face memory from the learning task. Face space models describe one way in which such reactivation-induced updating could occur (Valentine et al., 2016). These models suggest that individual faces are represented as having a unique location in a multidimensional mental face space, with a degree of error surrounding the location. Representational errors may lead to mistaken recognition of other faces due to information overlap between stored memories and incoming visual perception. When distractor faces 
were shown during refresh, their representations may have sometimes overlapped with stored target memories causing memory reactivation and leading to those distractors to be recognised as targets. Such reactivation in response to partial cues may have updated the target memories by biasing them towards the distractor location in face space, consistent with the view that face space is a dynamic system where face representations are updated to facilitate recognition (Rhodes, 2017). This plastic nature of face space could explain why face recognition induces updating without requiring reconstructive recollection of associations between different items and features that form a complex event memory.

However, although we are interpreting our findings as consistent with accounts whereby active retrieval attempts are more likely to reactive stored memories and thereby modify them (Bridge \& Voss, 2014; Dudai \& Eisenberg, 2004; Lee et al., 2017; Scully et al., 2017; St Jacques et al., 2013; St Jacques \& Schacter, 2013), we cannot show directly that memory reactivation differences between tasks account for our findings. In the testing effect literature, multiple potential mechanisms for why retrieval facilitates subsequent memory have been proposed, such as transfer appropriate processing and context reinstatement effects (reviewed in Karpicke, 2017), and these accounts could also be applied to biases that are induced by retrieval. Other potential explanations for how retrieval attempts could have enhanced distractor encoding are also possible. For example, participants may have attended more to selected faces in the recognition task than the control tasks due to particularly high difficulty when making recognition decisions, which in turn could have enhanced the encoding of falsely recognised distractor faces. We also cannot conclude that original target face memory traces were directly changed by recognition attempts, since distractor faces could have been encoded as separate memory traces (in line with e.g. Nadel et al., 2000) that subsequently caused those distractors to be more strongly recognised than targets. Future research is required to distinguish between these accounts, for example by measuring whether 
brain activity markers of memory reactivation and encoding during erroneous recognition of distractor faces predict subsequent biases ( $c f$. Bridge \& Paller, 2012; Bridge \& Voss, 2014; St Jacques et al., 2013; see also van den Honert et al., 2016)

Even though the exact mechanism underlying recognition-induced updating of face memories are still to be determined, our findings have implications for applied settings where it is important to know the reliability of eyewitness memory across repeated identification attempts. The 'commitment effect' has shown that eyewitnesses are likely to select the same face across multiple identification attempts following a crime, even when the identified face is not of the actual suspect, suggesting that recognition attempts also induce updating in real life settings (Lin et al., 2019; Steblay \& Dysart, 2016). Relatedly, research has found that errors introduced while trying to remember a suspect's face to create a facial composite can distort memories, but in some studies equivalent negative effects were also seen when people were passively exposed to those errors by viewing a composite face created by someone else (Topp-Manriquez et al., 2016). In contrast, our results suggest that face memory distortions were particularly enhanced following intentional recognition attempts compared to other types of exposure to erroneous face information. Complicating the picture further, other studies have found that creating facial composites can have positive or null net effects (Davis et al., 2014; Tredoux et al., 2020) on later recognition memory. Although these results may seem conflicting, they are consistent with the view that retrieval attempts induce updating, but such updating can have both positive and negative consequences for later memory depending on the accuracy of retrieval (e.g. Marsh et al., 2007). Therefore, repeated identification procedures are unlikely to have consistent effects on later face recognition, since such effects are likely to vary across situations, individuals and the particular face memory being tested (see discussion in Tredoux et al., 2020). 
In order to explain when recognition-induced updating occurs in applied eyewitness procedures, future research should use photographs of real human faces that include external features (such as hair), and also vary the facial images used between tests to better capture the natural variability of faces (Burton et al., 2016). This research should also adapt other aspects of the design to be closer to real eyewitness testimony procedures, such as including longer time delays between initial encoding and subsequent recognition attempts, presenting faces with bodies, and employ heightened emotional contexts to mimic a criminal event. It will also be important to determine whether different populations show similar degrees of recognition-induced updating of face memories, and to delineate other factors that modulate updating. Our participant samples were typical of Psychology undergraduate students in terms of being predominantly female young adults, so future research should investigate if updating occurs to a similar extent for other groups of people (e.g. males, older adults, children), and whether face memory updating is influenced by biases that are known to influence veridical face recognition (such as different kinds of own-group biases, Mukudi \& Hills, 2019).

In conclusion, the results of three experiments suggest that recognition attempts induce updating of face memories, and does so to a greater extent than other types of exposure to erroneous face information. These findings advance our understanding of memory updating by showing that item memories with minimal semantic associations can be distorted by erroneous retrieval attempts, and that such updating can occur even when the task does not require reconstructive recollection. Our results suggest that being in an intentional retrieval "mode" during face recognition enhances learning of new information in the retrieval environment, which is likely adaptive for keeping our memories up to date but can also cause memory distortions. 


\section{References}

Berntsen, D. (1996). Involuntary Autobiographical Memories. Applied Cognitive Psychology, 10(5), 435-454. https://doi.org/10.1002/(SICI)1099-0720(199610)10:5<435::AIDACP408>3.0.CO;2-L

Bird, C. M. (2017). The role of the hippocampus in recognition memory. Cortex; a Journal Devoted to the Study of the Nervous System and Behavior, 93, 155-165. https://doi.org/10.1016/j.cortex.2017.05.016

Bjork, E. L., Soderstrom, N. C., \& Little, J. L. (2015). Can Multiple-Choice Testing Induce Desirable Difficulties? Evidence from the Laboratory and the Classroom. The American Journal of Psychology, 128(2), 229-239. https://doi.org/10.5406/amerjpsyc.128.2.0229

Bridge, D. J., \& Paller, K. A. (2012). Neural Correlates of Reactivation and RetrievalInduced Distortion. Journal of Neuroscience, 32(35), 12144-12151.

Bridge, D. J., \& Voss, J. L. (2014). Active retrieval facilitates across-episode binding by modulating the content of memory. Neuropsychologia, 63, 154-164. https://doi.org/10.1016/j.neuropsychologia.2014.08.024

Burton, A. M., Kramer, R. S. S., Ritchie, K. L., \& Jenkins, R. (2016). Identity From Variation: Representations of Faces Derived From Multiple Instances. Cognitive Science, 40(1), 202-223. https://doi.org/10.1111/cogs.12231

Carpenter, S. K., \& Kelly, J. W. (2012). Tests enhance retention and transfer of spatial learning. Psychonomic Bulletin \& Review, 19(3), 443-448. https://doi.org/10.3758/s13423-012-0221-2

Coverdale, M. E., \& Nairne, J. S. (2019). The mnemonic effect of choice. Psychonomic Bulletin \& Review, 26(4), 1310-1316. https://doi.org/10.3758/s13423-019-01575-z 
Davis, J. P., Gibson, S., \& Solomon, C. (2014). The Positive Influence of Creating a Holistic Facial Composite on Video Line-up Identification. Applied Cognitive Psychology, 28(5), 634-639. https://doi.org/10.1002/acp.3045

Dienes, Z. (2011). Bayesian Versus Orthodox Statistics: Which Side Are You On? Perspectives on Psychological Science, 6(3), 274-290. https://doi.org/10.1177/1745691611406920

Duchaine, B., \& Yovel, G. (2015). A Revised Neural Framework for Face Processing. Annual Review of Vision Science, 1, 393-416. https://doi.org/10.1146/annurev-vision082114-035518

Dudai, Y., \& Eisenberg, M. (2004). Rites of passage of the engram: Reconsolidation and the lingering consolidation hypothesis. Neuron, 44(1), 93-100. https://doi.org/10.1016/j.neuron.2004.09.003

Ferreira, C. S., \& Wimber, M. (2021). The testing effect for visual materials depends on preexisting knowledge. PsyArXiv. https://doi.org/10.31234/osf.io/t6jdn

Gao, C., Rosburg, T., Hou, M., Li, B., Xiao, X., \& Guo, C. (2016). The role of retrieval mode and retrieval orientation in retrieval practice: Insights from comparing recognition memory testing formats and restudying. Cognitive, Affective, \& Behavioral Neuroscience, 16(6), 977-990. https://doi.org/10.3758/s13415-016-0446-z

Gibson, S. J., Solomon, C. J., \& Bejarano, A. P. (2003). Synthesis of Photographic Quality Facial Composites using Evolutionary Algorithms. Procedings of the British Machine Vision Conference 2003, 23.1-23.10. https://doi.org/10.5244/C.17.23

Haxby, J. V., \& Gobbini, M. I. (2011). Distributed Neural Systems for Face Perception. Oxford University Press. https://doi.org/10.1093/oxfordhb/9780199559053.013.0006 
Horner, A. J., Bisby, J. A., Bush, D., Lin, W.-J., \& Burgess, N. (2015). Evidence for holistic episodic recollection via hippocampal pattern completion. Nature Communications, 6(1), 7462. https://doi.org/10.1038/ncomms8462

Hupbach, A., Gomez, R., Hardt, O., \& Nadel, L. (2007). Reconsolidation of episodic memories: A subtle reminder triggers integration of new information. Learning \& Memory, 14(1-2), 47-53. https://doi.org/10.1101/lm.365707

Kanwisher, N., McDermott, J., \& Chun, M. M. (1997). The Fusiform Face Area: A Module in Human Extrastriate Cortex Specialized for Face Perception. Journal of Neuroscience, 17(11), 4302-4311. https://doi.org/10.1523/JNEUROSCI.17-1104302.1997

Karpicke, J. D. (2017). Retrieval-Based Learning: A Decade of Progress. In Learning and Memory: A Comprehensive Reference (pp. 487-514). Elsevier. https://doi.org/10.1016/B978-0-12-809324-5.21055-9

Karpicke, J. D., \& Zaromb, F. M. (2010). Retrieval mode distinguishes the testing effect from the generation effect. Journal of Memory and Language, 62(3), 227-239. https://doi.org/10.1016/j.jml.2009.11.010

Lee, J. L. C., Nader, K., \& Schiller, D. (2017). An Update on Memory Reconsolidation Updating. Trends in Cognitive Sciences, 21(7), 531-545. https://doi.org/10.1016/j.tics.2017.04.006

Lin, W., Strube, M. J., \& Roediger, H. L. (2019). The effects of repeated lineups and delay on eyewitness identification. Cognitive Research: Principles and Implications, 4(1), 16. https://doi.org/10.1186/s41235-019-0168-1

Little, J. L., Bjork, E. L., Bjork, R. A., \& Angello, G. (2012). Multiple-Choice Tests Exonerated, at Least of Some Charges: Fostering Test-Induced Learning and 
Avoiding Test-Induced Forgetting. Psychological Science, 23(11), 1337-1344. https://doi.org/10.1177/0956797612443370

Liu, Y., Rosburg, T., Gao, C., Weber, C., \& Guo, C. (2017). Differentiation of subsequent memory effects between retrieval practice and elaborative study. Biological Psychology, 127, 134-147. https://doi.org/10.1016/j.biopsycho.2017.05.010

Marsh, E. J., Roediger, H. L., Bjork, R. A., \& Bjork, E. L. (2007). The memorial consequences of multiple-choice testing. Psychonomic Bulletin \& Review, 14(2), 194199. https://doi.org/10.3758/BF03194051

Mist, J. J., Gibson, S. J., \& Solomon, C. J. (2015). Comparing Evolutionary Operators, Search Spaces, and Evolutionary Algorithms in the Construction of Facial Composites. Informatica, 39(2), Article 2. http://www.informatica.si/index.php/informatica/article/view/830

Mukudi, P. B. L., \& Hills, P. J. (2019). The combined influence of the own-age, -gender, and -ethnicity biases on face recognition. Acta Psychologica, 194, 1-6. https://doi.org/10.1016/j.actpsy.2019.01.009

Murty, V. P., DuBrow, S., \& Davachi, L. (2015). The simple act of choosing influences declarative memory. The Journal of Neuroscience: The Official Journal of the Society for Neuroscience, 35(16), 6255-6264. https://doi.org/10.1523/JNEUROSCI.418114.2015

Nadel, L., Samsonovich, A., Ryan, L., \& Moscovitch, M. (2000). Multiple trace theory of human memory: Computational, neuroimaging, and neuropsychological results. Hippocampus, 10(4), 352-368. https://doi.org/10.1002/10981063(2000)10:4<352::AID-HIPO2>3.0.CO;2-D

Oosterhof, N. N., \& Todorov, A. (2008). The functional basis of face evaluation. Proceedings of the National Academy of Sciences, 105(32), 11087-11092. 
Ranganath, C., \& Ritchey, M. (2012). Two cortical systems for memory-guided behaviour. Nature Reviews. Neuroscience, 13(10), 713-726. https://doi.org/10.1038/nrn3338 Rhodes, G. (2017). Adaptive Coding and Face Recognition. Current Directions in Psychological Science, 26(3), 218-224. https://doi.org/10.1177/0963721417692786

Richardson-klavehn, A., Gardiner, J. M., \& Java, R. I. (1994). Involuntary conscious memory and the method of opposition. Memory, 2(1), 1-29. https://doi.org/10.1080/09658219408251490

Roediger, H. L., \& Butler, A. C. (2011). The critical role of retrieval practice in long-term retention. Trends in Cognitive Sciences, 15(1), 20-27. https://doi.org/10.1016/j.tics.2010.09.003

Roediger, H. L., \& Karpicke, J. D. (2006). Test-Enhanced Learning: Taking Memory Tests Improves Long-Term Retention. Psychological Science, 17(3), 249-255. https://doi.org/10.1111/j.1467-9280.2006.01693.x

Roediger III, H. L., \& Marsh, E. J. (2005). The Positive and Negative Consequences of Multiple-Choice Testing. Journal of Experimental Psychology: Learning, Memory, and Cognition, 31(5), 1155-1159. https://doi.org/10.1037/0278-7393.31.5.1155

Rolls, E. T. (2016). Pattern separation, completion, and categorisation in the hippocampus and neocortex. Neurobiology of Learning and Memory, 129, 4-28. https://doi.org/10.1016/j.nlm.2015.07.008

Rowland, C. A. (2014). The effect of testing versus restudy on retention: A meta-analytic review of the testing effect. Psychological Bulletin, 140(6), 1432-1463. https://doi.org/10.1037/a0037559

Rugg, M. D., \& Wilding, E. L. (2000). Retrieval processing and episodic memory. Trends in Cognitive Sciences, 4(3), 108-115. https://doi.org/10.1016/S1364-6613(00)01445-5 
Schacter, D. L., Guerin, S. A., \& Jacques, P. L. S. (2011). Memory distortion: An adaptive perspective. Trends in Cognitive Sciences, 15(10), 467-474. https://doi.org/10.1016/j.tics.2011.08.004

Scully, I. D., Napper, L. E., \& Hupbach, A. (2017). Does reactivation trigger episodic memory change? A meta-analysis. Neurobiology of Learning and Memory, 142, 99 107. https://doi.org/10.1016/j.nlm.2016.12.012

Solomon, C. J., Gibson, S. J., \& Mist, J. J. (2013). Interactive evolutionary generation of facial composites for locating suspects in criminal investigations. Applied Soft Computing, 13(7), 3298-3306. https://doi.org/10.1016/j.asoc.2013.02.010

Sporer, S. L., Tredoux, C. G., Vredeveldt, A., Kempen, K., \& Nortje, A. (2020). Does exposure to facial composites damage eyewitness memory? A comprehensive review. Applied Cognitive Psychology, 34(5), 1166-1179. https://doi.org/10.1002/acp.3705

St Jacques, P. L., Olm, C., \& Schacter, D. L. (2013). Neural mechanisms of reactivationinduced updating that enhance and distort memory. Proceedings of the National Academy of Sciences of the United States of America, 110(49), 19671-19678. https://doi.org/10.1073/pnas.1319630110

St Jacques, P. L., \& Schacter, D. L. (2013). Modifying memory: Selectively enhancing and updating personal memories for a museum tour by reactivating them. Psychological Science, 24(4), 537-543. https://doi.org/10.1177/0956797612457377

Steblay, N. K., \& Dysart, J. E. (2016). Repeated eyewitness identification procedures with the same suspect. Journal of Applied Research in Memory and Cognition, 5(3), 284289. https://doi.org/10.1016/j.jarmac.2016.06.010

Topp-Manriquez, L. D., McQuiston, D., \& Malpass, R. S. (2016). Facial composites and the misinformation effect: How composites distort memory. Legal and Criminological Psychology, 21(2), 372-389. https://doi.org/10.1111/lcrp.12054 
Tredoux, C. G., Sporer, S. L., Vredeveldt, A., Kempen, K., \& Nortje, A. (2020). Does constructing a facial composite affect eyewitness memory? A research synthesis and meta-analysis. Journal of Experimental Criminology. https://doi.org/10.1007/s11292020-09432-z

Treisman, A. M., \& Gelade, G. (1980). A feature-integration theory of attention. Cognitive Psychology, 12(1), 97-136. https://doi.org/10.1016/0010-0285(80)90005-5

Tulving, E. (1983). Elements of Episodic Memory. Oxford University Press.

Valentine, T., Lewis, M. B., \& Hills, P. J. (2016). Face-Space: A Unifying Concept in Face Recognition Research. Quarterly Journal of Experimental Psychology, 69(10), 19962019. https://doi.org/10.1080/17470218.2014.990392

van den Honert, R. N., McCarthy, G., \& Johnson, M. K. (2016). Reactivation during encoding supports the later discrimination of similar episodic memories. Hippocampus, 26(9), 1168-1178. https://doi.org/10.1002/hipo.22598

Watanabe, T., \& Soraci, S. A. (2004). The self-choice effect from a multiple-cue perspective. Psychonomic Bulletin \& Review, 11(1), 168-172. https://doi.org/10.3758/BF03206478

Winograd, E. (1981). Elaboration and distinctiveness in memory for faces. Journal of Experimental Psychology: Human Learning and Memory, 7(3), 181-190. https://doi.org/10.1037/0278-7393.7.3.181

Ye, Z., Shi, L., Li, A., Chen, C., \& Xue, G. (2020). Retrieval practice facilitates memory updating by enhancing and differentiating medial prefrontal cortex representations. ELife, 9, e57023. https://doi.org/10.7554/eLife.57023

Yonelinas, A. P. (2002). The Nature of Recollection and Familiarity: A Review of 30 Years of Research. Journal of Memory and Language, 46(3), 441-517. https://doi.org/10.1006/jmla.2002.2864 